\title{
Nondegenerate two-beam coupling in Kerr nonlinear photonic crystals
}

\author{
Ping Xie and Zhao-Qing Zhang \\ Department of Physics and Institute of Nano Science and Technology (INST), The Hong Kong University of Science and Technology, \\ Clear Water Bay, Hong Kong, China
}

(Received 22 September 2004; revised manuscript received 23 May 2005; published 14 September 2005)

\begin{abstract}
We show that the energy-transfer efficiency by nondegenerate two-beam coupling in a one-dimensional Kerr-nonlinear superlattice can be enhanced by several orders of magnitude as compared with that in a homogeneous medium of the same nonlinearity and length. This significant enhancement utilizes the strong localized field at the band-edge state, two-frequency localized state, or defect state. Due to the intensityinduced index modulation, the bistability is observed, and because of the energy transfer between different wavelength components, the tristability behavior is induced.
\end{abstract}

DOI: 10.1103/PhysRevE.72.036607

PACS number(s): 42.70.Qs, 42.65.Pc, 63.20.Pw

\section{INTRODUCTION}

Nonlinear photonic crystals have recently attracted considerable attention [1]. The combination of both photonic crystals and nonlinear optics has opened up an area of research for both science and technology. In $\chi^{(2)}$ nonlinear photonic crystals, most of the works have focused on the enhancement of the second-harmonic or sum-frequency generation by use of the simultaneous availability of field localization and phase-matching conditions at the band edge [2].

For $\chi^{(3)}$ nonlinear photonic crystals, the works have focused mainly on the effects related to the refractive-index change of the nonlinear layers caused by the light intensity via Kerr effect. For example, by changing the light intensity at frequency in the pass band, the band-edge frequency can be dynamically tuned. Based on this mechanism, the optical limiting and switching in one-dimensional (1D) nonlinear photonic crystals have been studied [3]. By changing the light intensity at the frequency near a band edge [4] or a defect mode [5], the frequency of the band-edge state or defect mode shifts, leading to bistability phenomena. For frequencies inside the gap, the formation of the $\chi^{(3)}$-induced self-organized localized state with a single-frequency component, which is called single-frequency gap soliton [6-8], and that with multifrequency components, which is called multi-frequency gap soliton [9], have been demonstrated.

Recently, much attention has been paid to utilize the properties of field localization at defect states or gap-soliton states to enhance the third-order nonlinear optical effects. For example, the significant enhancement of the thirdharmonic generation by use of both field localization and phase-matching conditions near the band edge or inside the gap has been studied [10]. Furthermore, it has been shown that the efficiency of the phase conjugation generation in degenerate four-wave mixing could be enhanced greatly by utilizing the strong field localization of a gap soliton or a defect state [11].

In this work we demonstrate that the great enhancement of the energy-transfer efficiency in the $\chi^{(3)}$-related nondegenerate two-beam coupling can also be realized by utilizing the self-organized localized states and the shifted defect modes induced by the incident waves. This presents a way toward high-efficiency, microsized optical devices for optical signal amplification and optical interconnection.

\section{THEORY}

Consider the two-beam coupling in a 1D Kerr-nonlinear superlattice using the geometry shown in Fig. 1. In this geometry, the lossless nonlinear superlattice composes of $N$ unit cells. One layer in each unit cell is linear, with the refractive index $n_{01}$ and width $l_{1}$. The other layer is nonlinear, characterized by the usual, weak-field refractive index $n_{02}$ and a Kerr coefficient $n_{2}$. The width of the nonlinear layer is $l_{2}$. Two beams $\widetilde{E}_{1}$ and $\widetilde{E}_{2}$ are incident upon the superlattice with incident angles $\theta_{10}$ and $\theta_{20}$, respectively. Beam $\widetilde{E}_{1}$ has frequency $\omega_{1}$ and beam $\widetilde{E}_{2}$ has frequency $\omega_{2}$. Because of reflections at each interface of two layers, in each layer, there appear two additional beams, $\widetilde{E}_{-1}$ and $\widetilde{E}_{-2}$, which are the mirror reflections of $\widetilde{E}_{1}$ and $\widetilde{E}_{2}$, respectively. Owing to the nonlinear interaction in the nonlinear layers, energy can exchange between beams $\widetilde{E}_{1}$ and $\widetilde{E}_{-1}$ and between beams $\widetilde{E}_{2}$ and $\widetilde{E}_{-2}$. For simplicity, all waves are assumed to be $E$ polarized with the electric field perpendicular to the plane of incidence.

For plane waves, we represent the four beams in the layered medium by

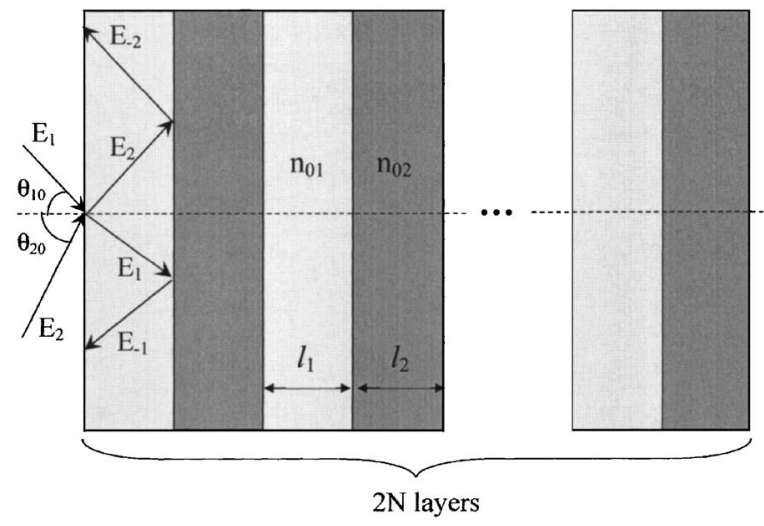

FIG. 1. Geometry of nondegenerate two-beam coupling in a 1D photonic crystal with Kerr nonlinearity. Beam $E_{1}$ with frequency $\omega_{1}$ and beam $E_{2}$ with frequency $\omega_{2}$ are two input beams. $E_{-1}$ and $E_{-2}$ are the mirror reflections of $E_{1}$ and $E_{2}$, respectively. 


$$
\begin{gathered}
\widetilde{E}_{\alpha}(\vec{r}, t)=E_{\alpha}(\vec{r}) e^{-i \omega_{1} t}+\text { c.c. }=A_{\alpha}(z) e^{i \overrightarrow{k_{\alpha}} \cdot \vec{r}-i \omega_{1} t}+\text { c.c. } \\
(\alpha=1,-1), \\
\widetilde{E}_{\alpha}(\vec{r}, t)=E_{\alpha}(\vec{r}) e^{-i \omega_{2} t}+\text { c.c. }=A_{\alpha}(z) e^{i \vec{k}_{\alpha} \cdot \vec{r}-i \omega_{2} t}+\text { c.c. } \\
(\alpha=2,-2) .
\end{gathered}
$$

The total intensity distribution, $\left.I=\left\langle\widetilde{E}_{1}+\widetilde{E}_{2}+\widetilde{E}_{-1}+\widetilde{E}_{-2}\right)^{2}\right\rangle_{t}$, is hence given by

$$
\begin{aligned}
I= & \left|A_{1}\right|^{2}+\left|A_{2}\right|^{2}+\left|A_{-1}\right|^{2}+\left|A_{-2}\right|^{2}+A_{1} A_{-1}^{*} e^{i\left(\vec{k}_{1}-\vec{k}_{-1}\right) \cdot \vec{r}} \\
& +A_{2} A_{-2}^{*} e^{i\left(\vec{k}_{2}-\vec{k}_{-2}\right) \vec{r}}+A_{1} A_{2}^{*} e^{i\left(\vec{k}_{1}-\vec{k}_{2}\right) \vec{r}} e^{-i \delta t}+A_{1} A_{-2}^{*} e^{i\left(\vec{k}_{1}-\vec{k}_{-2}\right) \vec{r}} e^{-i \delta t} \\
& +A_{-1} A_{2}^{*} e^{i\left(\vec{k}_{-1}-\vec{k}_{2}\right) \vec{r}} e^{-i \delta t}+A_{-1} A_{-2}^{*} e^{i\left(\vec{k}_{-1}-\vec{k}_{-2}\right) \vec{r}} e^{-i \delta t}+\text { c.c. }
\end{aligned}
$$

where the angular bracket represents an average over a time interval of many optical periods and $\delta=\omega_{1}-\omega_{2}$ is the frequency detuning. In a Kerr medium, the nonlinear part of the refractive index obeys a Debye relaxation equation of the form [12]

$$
\tau \frac{d n_{N L}}{d t}+n_{N L}=n_{2} I,
$$

where $\tau$ is the response time of the Kerr effect. Substituting Eq. (2) into Eq. (3) and then solving Eq. (3) we finally obtain

$$
\begin{aligned}
n_{N L}= & n_{2}\left[\left|A_{1}\right|^{2}+\left|A_{2}\right|^{2}+\left|A_{-1}\right|^{2}+\left|A_{-2}\right|^{2}+A_{1} A_{-1}^{*} e^{i\left(\vec{k}_{1}-\vec{k}_{-1}\right) \vec{r}}\right. \\
& +A_{2} A_{-2}^{*} e^{i\left(\vec{k}_{2}-\vec{k}_{-2}\right) \vec{r}}+\frac{A_{1} A_{2}^{*} e^{i\left(\vec{k}_{1}-\vec{k}_{2}\right) r} e^{-i \delta t}}{1-i \delta \tau} \\
& +\frac{A_{1} A_{-2}^{*} e^{i\left(\vec{k}_{1}-\vec{k}_{-2}\right) \vec{r}} e^{-i \delta t}}{1-i \delta \tau}+\frac{A_{-1} A_{2}^{*} e^{i\left(\vec{k}_{-1}-\vec{k}_{2}\right) \vec{r}} e^{-i \delta t}}{1-i \delta \tau} \\
& \left.+\frac{A_{-1} A_{-2}^{*} e^{i\left(\vec{k}_{-1}-\vec{k}_{-2}\right) \vec{r}} e^{-i \delta t}}{1-i \delta \tau}+\text { c.c. }\right] .
\end{aligned}
$$

Substituting Eqs. (1a) and (1b) and $n^{2}=n_{02}^{2}+2 n_{02} n_{N L}$ into

$$
\nabla^{2} \widetilde{E}-\frac{n^{2}}{c^{2}} \frac{\partial^{2} \widetilde{E}}{\partial t^{2}}=0
$$

and making the slowly varying-amplitude approximation, we obtain

$$
\begin{gathered}
\frac{d A_{\alpha}}{d z}=i \kappa_{\alpha} A_{\alpha}, \quad(\alpha=1,2), \\
\frac{d A_{\alpha}}{d z}=-i \kappa_{\alpha} A_{\alpha}, \quad(\alpha=-1,-2),
\end{gathered}
$$

where

$$
\begin{aligned}
\kappa_{1}= & \frac{n_{2} \omega_{1}}{c \cos \theta_{1}}\left(\left|A_{1}\right|^{2}+\left|A_{2}\right|^{2}+2\left|A_{-1}\right|^{2}+\left|A_{-2}\right|^{2}\right) \\
& +\frac{n_{2} \omega_{2}}{c \cos \theta_{1}} \frac{\left|A_{2}\right|^{2}+\left|A_{-2}\right|^{2}}{1-i \delta \tau},
\end{aligned}
$$

$$
\begin{aligned}
\kappa_{2}= & \frac{n_{2} \omega_{2}}{c \cos \theta_{2}}\left(\left|A_{1}\right|^{2}+\left|A_{2}\right|^{2}+\left|A_{-1}\right|^{2}+2\left|A_{-2}\right|^{2}\right) \\
& +\frac{n_{2} \omega_{1}}{c \cos \theta_{2}} \frac{\left|A_{1}\right|^{2}+\left|A_{-1}\right|^{2}}{1+i \delta \tau}, \\
\kappa_{-1}= & \frac{n_{2} \omega_{1}}{c \cos \theta_{1}}\left(2\left|A_{1}\right|^{2}+\left|A_{2}\right|^{2}+\left|A_{-1}\right|^{2}+\left|A_{-2}\right|^{2}\right) \\
& +\frac{n_{2} \omega_{2}}{c \cos \theta_{1}} \frac{\left|A_{2}\right|^{2}+\left|A_{-2}\right|^{2}}{1-i \delta \tau}, \\
\kappa_{-2}= & \frac{n_{2} \omega_{2}}{c \cos \theta_{2}}\left(\left|A_{1}\right|^{2}+2\left|A_{2}\right|^{2}+\left|A_{-1}\right|^{2}+\left|A_{-2}\right|^{2}\right) \\
& +\frac{n_{2} \omega_{1}}{c \cos \theta_{2}} \frac{\left|A_{1}\right|^{2}+\left|A_{-1}\right|^{2}}{1+i \delta \tau} .
\end{aligned}
$$

Here, $\theta_{1}$ and $\theta_{2}$ are propagation angles of $\widetilde{E}_{1}$ and $\widetilde{E}_{2}$ inside the nonlinear medium. It should be mentioned that the imaginary parts of $\kappa_{\alpha}$ in Eqs. (6a)-(6d) represent energy exchange between frequency components, which is peaked when $|\delta \tau|$ $=1$. In the case of a homogeneous medium, where $\widetilde{E}_{-1}$ $=\widetilde{E}_{-2}=0$, it is easy to show that the total energy current density of the two components along the $z$ direction is a constant, independent of the exchange.

In order to solve Eqs. (5a), (5b), and (6a)-(6d), we divide each nonlinear layer into $M$ sublayers and assume the values of $\kappa_{\alpha}(\alpha=1,2,-1,-2)$ in each sublayer to be constants and equal to their mean values in the sublayer. In our calculations, $M$ is taken so that a convergent result is reached. After solving Eqs. (5a) and (5b) in each sublayer, we obtain

$$
\begin{gathered}
A_{\alpha}\left(l_{s, i}\right)=e^{i \kappa_{\alpha} l_{s, i} A_{\alpha}(0), \quad(\alpha=1,2),} \\
A_{\alpha}\left(l_{s, i}\right)=e^{-i \kappa_{\alpha} l_{s, i}} A_{\alpha}(0), \quad(\alpha=-1,-2),
\end{gathered}
$$

where $A_{\alpha} 0$ denotes the field amplitude at one boundary and $A_{\alpha}\left(l_{s, i}\right)$ at the other boundary of the sublayer $i$ of width $l_{s, i}$ in the $s$ layer.

From Eqs. (1a), (1b), (7a), and (7b), we have the following relation:

$$
\begin{gathered}
E_{\alpha}\left(l_{s, i}\right)=e^{i \Delta_{\alpha}} E_{\alpha}(0), \quad(\alpha=1,2), \\
E_{\alpha}\left(l_{s, i}\right)=e^{-i \Delta_{\alpha}} E_{\alpha}(0), \quad(\alpha=-1,-2),
\end{gathered}
$$

where $E_{\alpha}(0)$ denotes the field at one boundary and $E_{\alpha}\left(l_{s, i}\right)$ at the other boundary of the sublayer $i$ of width $l_{s, i}$ in the $s$ layer, $\Delta_{\beta}=l_{s, i}\left(n_{0}^{(s, i)} k_{\beta} \cos \theta_{1}^{(s, i)}+\kappa_{\beta}\right), \quad(\beta=1,-1), \quad$ and $\Delta_{\beta}$ $=l_{s, i}\left(n_{0}^{(s, i)} k_{\beta} \cos \theta_{2}^{(s, i)}+\kappa_{\beta}\right),(\beta=2,-2)$. Here, $n_{0}^{(s, i)}$ denotes the weak-field refractive index of the sublayer, $\theta_{1}^{(s, i)}$ and $\theta_{2}^{(s, i)}$ are propagation angles of $\widetilde{E}_{1}$ and $\widetilde{E}_{2}$, respectively, in the sublayer. From the continuity of both $E$ and $H$ (i.e., the derivative of $E$ ) at the interface of two sublayers, we have the following relation: 


$$
\begin{aligned}
& {\left[\begin{array}{c}
E_{1}(0) \\
E_{-1}(0)
\end{array}\right]^{(s, i)}=\frac{1}{2}\left[\begin{array}{ll}
1+S_{1} & 1-S_{1} \\
1-S_{1} & 1+S_{1}
\end{array}\right]\left[\begin{array}{c}
E_{1}\left(l_{s, i-1}\right) \\
E_{-1}\left(l_{s, i-1}\right)
\end{array}\right]^{(s, i-1)},} \\
& {\left[\begin{array}{c}
E_{2}(0) \\
E_{-2}(0)
\end{array}\right]^{(s, i)}=\frac{1}{2}\left[\begin{array}{ll}
1+S_{2} & 1-S_{2} \\
1-S_{2} & 1+S_{2}
\end{array}\right]\left[\begin{array}{c}
E_{2}\left(l_{s, i-1}\right) \\
E_{-2}\left(l_{s, i-1}\right)
\end{array}\right]^{(s, i-1)},}
\end{aligned}
$$

where $S_{1}=\left[n_{0}^{(s, i-1)} \cos \theta_{1}^{(s, i-1)}\right] /\left[n_{0}^{(s, i)} \cos \theta_{1}^{(s, i)}\right] \quad$ and $S_{2}$ $=\left[n_{0}^{(s, i-1)} \cos \theta_{2}^{(s, i-1)}\right] /\left[n_{0}^{(s, i)} \cos \theta_{2}^{(s, i)}\right]$. Combining Eqs. (8a), (8b), (9a), and (9b) we get

$$
\begin{aligned}
& {\left[\begin{array}{c}
E_{1}\left(l_{s, i}\right) \\
E_{-1}\left(l_{s, i}\right)
\end{array}\right]^{(s, i)}=t_{1}^{(s, i)}\left[\begin{array}{c}
E_{1}\left(l_{s, i-1}\right) \\
E_{-1}\left(l_{s, i-1}\right)
\end{array}\right]{ }^{(s, i-1)},} \\
& t_{1}^{(s, i)}=\frac{1}{2}\left[\begin{array}{cc}
\left(1+S_{1}\right) e^{i \Delta_{\alpha}} & \left(1-S_{1}\right) e^{i \Delta_{\alpha}} \\
\left(1-S_{1}\right) e^{-i \Delta_{\beta}} & \left(1+S_{1}\right) e^{-i \Delta_{\beta}}
\end{array}\right],
\end{aligned}
$$

and

$$
\begin{aligned}
& {\left[\begin{array}{c}
E_{2}\left(l_{s, i}\right) \\
E_{-2}\left(l_{s, i}\right)
\end{array}\right]^{(s, i)}=t_{2}^{(s, i)}\left[\begin{array}{c}
E_{2}\left(l_{s, i-1}\right) \\
E_{-2}\left(l_{s, i-1}\right)
\end{array}\right]^{(s, i-1)},} \\
& t_{2}^{(s, i)}=\frac{1}{2}\left[\begin{array}{cc}
\left(1+S_{2}\right) e^{i \Delta_{\alpha}} & \left(1-S_{2}\right) e^{i \Delta_{\alpha}} \\
\left(1-S_{2}\right) e^{-i \Delta_{\beta}} & \left(1+S_{2}\right) e^{-i \Delta_{\beta}}
\end{array}\right] .
\end{aligned}
$$

For the linear layer, we take $n_{2}=0$ and $M=1$. From the products of these matrices, $T_{1}=\prod_{i=1}^{N(M+1)} t_{1}^{(s, i)}$ and $T_{2}=\prod_{i=1}^{N(M+1)} t_{2}^{(s, i)}$, we obtain the transmission coefficients for $E_{1}$ and $E_{2}$ as

$$
\begin{aligned}
& \frac{E_{1}(L)}{E_{1}(0)}=\frac{T_{1}(1,1) T_{1}(2,2)-T_{1}(1,2) T_{1}(2,1)}{T_{1}(2,2)}, \\
& \frac{E_{2}(L)}{E_{2}(0)}=\frac{T_{2}(1,1) T_{2}(2,2)-T_{2}(1,2) T_{2}(2,1)}{T_{2}(2,2)},
\end{aligned}
$$

and the reflection coefficients as

$$
\begin{aligned}
& \frac{E_{-1}(0)}{E_{1}(0)}=-\frac{T_{1}(2,1)}{T_{1}(2,2)}, \\
& \frac{E_{-2}(0)}{E_{2}(0)}=-\frac{T_{2}(2,1)}{T_{2}(2,2)} .
\end{aligned}
$$

\section{NUMERICAL CALCULATION AND DISCUSSION}

The equations in Sec. II are solved numerically by using an iterative procedure described in Refs. [8,9]. We emphasize that all the solutions presented below are stable. In fact, if a solution is not stable in time, i.e., the solution is not a fixed attractor, the iterative procedure will never be convergent to a limiting result. For calculations, we consider a $\mathrm{CdS} / \mathrm{SiO}_{2}$ superlattice of $N$ unit cells, i.e., $N$ nonlinear layers and $N$ linear layers. $\mathrm{SiO}_{2}$ can be considered to be linear, with refractive index $n_{01}=1.45$ in the wavelength of $\lambda \approx 680 \mathrm{~nm}$. For the nonlinear CdS layers, $n_{02}=2.4, \chi^{(3)}=-10^{-10} \mathrm{esu}$, or
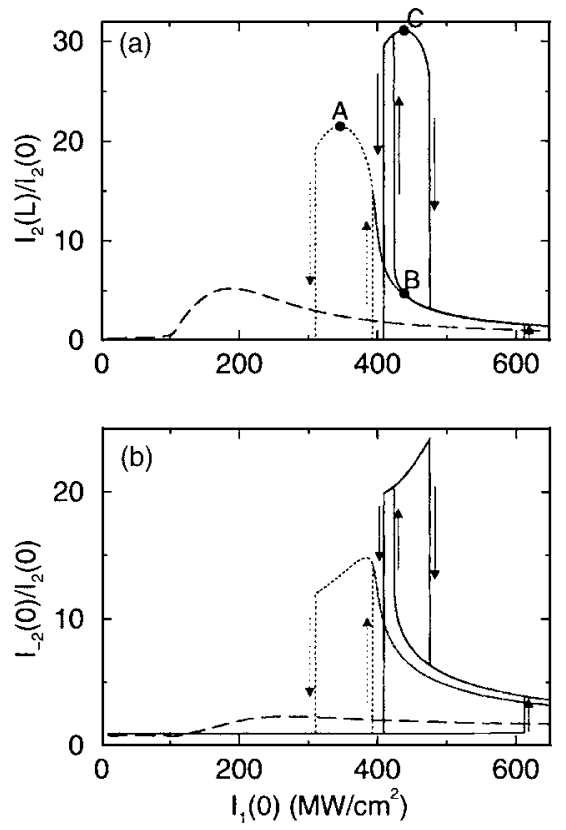

FIG. 2. $I_{2}(L) / I_{2}(0)$ (a) and $I_{-2}(0) / I_{2}(0)(\mathrm{b})$ as a function of $I_{1}(0)$ in the superlattice of $N=30$ unit cells. Dashed curves: $\lambda_{1}$ $=681.83 \mathrm{~nm}, \lambda_{2}=680.85 \mathrm{~nm}$. Dotted curves: $\lambda_{1}=681.45 \mathrm{~nm}, \lambda_{2}$ $=680.47 \mathrm{~nm}$. Solid curves: $\lambda_{1}=681.3 \mathrm{~nm}, \lambda_{2}=680.32 \mathrm{~nm}$.

equivalently, $\quad n_{2} \equiv 6 \pi \chi^{(3)} / n_{02}=-7.85 \times 10^{-10}$ esu, and $\tau$ $=250$ fs $[13]$.

Throughout this work we take the incident angles for the two input beams as $\theta_{10}=2^{\circ}$ and $\theta_{20}=7^{\circ}$.

\section{A. Enhancement of energy transfer by making use of gap localized state}

We first study the enhancement of energy-transfer efficiency by making use of the field localization at band-edge state or self-organized localized state inside the gap. To do so, we consider a superlattice of $N=30$ unit cells, i.e., 30 $\mathrm{SiO}_{2}$-linear layers and $30 \mathrm{CdS}$-nonlinear layers, with the width of each layer as $l_{1}=l_{2}=75 \mathrm{~nm}$. In the linear case, i.e., $n_{2}=0$, the transmission spectrum of a beam propagating through the superlattice with incident angles $\theta_{10}=2^{\circ}$ and $\theta_{20}=7^{\circ}$ gives the higher-wavelength band edges of the first band gap at $\lambda_{10}=682.27 \mathrm{~nm}$ and $\lambda_{20}=681.36 \mathrm{~nm}$, respectively. Since the Kerr nonlinearity $\left(n_{2}<0\right)$ induces the shift of the band edge or the formation of gap soliton [6-9], in order to make use of their localized fields we should choose the operating wavelengths $\lambda_{1}$ and $\lambda_{2}$ of the two input beams slightly smaller than $\lambda_{10}$ and $\lambda_{20}$, respectively. In Figs. 2(a) and 2(b) we show three typical results of the transmission $I_{2}(L) / I_{2}(0)$ and reflection $I_{-2}(0) / I_{2}(0)$, respectively, as a function of input pump intensity $I_{1}(0)$, with fixed $I_{2}(0)$ $=3.675 \mathrm{MW} / \mathrm{cm}^{2}$. Here we denote $I_{j}=\left|E_{j}\right|^{2}(j=1,2,-1,-2)$. Dashed curves are for $\lambda_{1}=681.83 \mathrm{~nm}$ and $\lambda_{2}=680.85 \mathrm{~nm}$, dotted curves for $\lambda_{1}=681.45 \mathrm{~nm}$ and $\lambda_{2}=680.47 \mathrm{~nm}$, and solid curves for $\lambda_{1}=681.3 \mathrm{~nm}$ and $\lambda_{2}=680.32 \mathrm{~nm}$. Note that the wavelengths used in Fig. 2 give $|\delta \tau|=\mid 2 \pi c \tau /\left(1 / \lambda_{1}\right.$ $\left.-1 / \lambda_{2}\right) \mid=0.99$, which is nearly unity. 


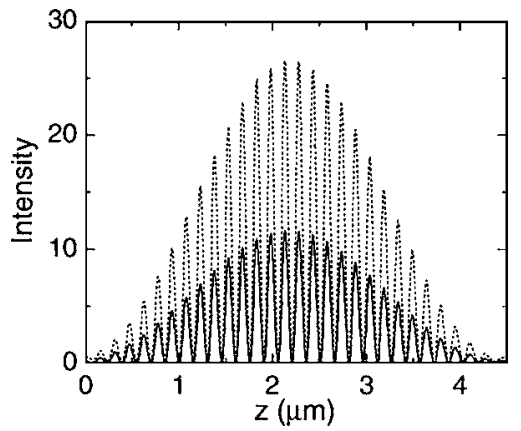

FIG. 3. Intensity distributions for components of wavelength $\lambda_{1}=681.45 \mathrm{~nm}$ (dotted curve) and wavelength $\lambda_{2}=680.47 \mathrm{~nm}$ (solid curve) at point $A$ of Fig. 2 . The intensity is normalized by $I_{1}(0)$.

When the operating wavelengths $\lambda_{1}$ and $\lambda_{2}$ are very near their band-edge wavelengths $\lambda_{10}$ and $\lambda_{20}, I_{2}(L) / I_{2}(0)$ [or $\left.I_{-2}(0) / I_{2}(0)\right]$ versus $I_{1}(0)$ has a single value, as shown by dashed curves. The maximum value of $I_{2}(L) / I_{2}(0)$ corresponds to the situation that the interacting beams are approximately operating at their shifted band-edge states. To compare with the result in a homogeneous medium we give a calculation of the gain in a medium of the same $n_{2}$ and length $L=30 l_{1}+30 l_{2}=4.5 \mu \mathrm{m}$. Now there exist only two interacting beams $\widetilde{E}_{1}$ and $\widetilde{E}_{2}$ inside the medium when neglecting the reflections at the input and output faces. From Eqs. (5a), (6a), and (6b) we can easily obtain $d I_{2} / d z$ $=\left(n_{2} \omega_{1}\right) /\left(c \cos \theta_{2}\right) / 2 \delta \tau /\left(1+\delta^{2} \tau^{2}\right) I_{1} I_{2}$. In the approximation of undepleted pump we have $I_{2}(L) \cong I_{2}(0) \exp (g L)$, where the gain coefficient $g=n_{2} \omega_{1} /\left(c \cos \theta_{2}\right) / 2 \delta \tau /\left(1+\delta^{2} \tau^{2}\right) I_{1}(0)$. Thus the increase of $I_{2}$ is $\left[I_{2}(L)-I_{2}(0)\right] / I_{2}(0) \approx 5.8 \times 10^{-3}$ for $I_{1}(0) \approx 187.6 \mathrm{MW} / \mathrm{cm}^{2}$ and $\lambda_{1}=2 \pi c / \omega_{1}=681 \mathrm{~nm}$. From Fig. 2 we note that the efficiency of energy transfer is enhanced by nearly three orders of magnitude.

From Fig. 2, we see that as the operating wavelengths $\lambda_{1}$ and $\lambda_{2}$ are moved into their band gaps the bistability of $I_{2}(L) / I_{2}(0)$ versus $I_{1}(0)$ occurs, as shown by dotted curves. This is similar to the transmission behavior of a single beam through the nonlinear superlattice, where the bistability is associated with the formation of a gap soliton [6-8]. The large value of $I_{2}(L) / I_{2}(0)$ in the dotted curves of Fig. 2 is due to the excitation of the self-organized field localizations for both $\lambda_{1}$ - and $\lambda_{2}$-wavelength components. This can be seen from the intensity distributions for both wavelength components at point $A$, as shown in Fig. 3. The localized fields for two wavelengths are induced by a common refractive-index modulation of the nonlinear layers.

From Fig. 2, it is interesting to note that, as the operating wavelengths $\lambda_{1}$ and $\lambda_{2}$ are further moved into their gaps, tristability of $I_{2}(L) / I_{2}(0)$ versus $I_{1}(0)$ occurs, as shown by solid curves. We verified that both the coupled localized fields for two wavelength components at point $B$ and those at point $C$ have single-envelope distributions, which are shown in Figs. 4(a) and 4(b), respectively. Figures 4(a) and 4(b) represent the two different stable states with different localized intensities for two wavelengths. The existence of two localized states in this case is a result of nonlinear energy exchange between the wavelength components. This is dif-
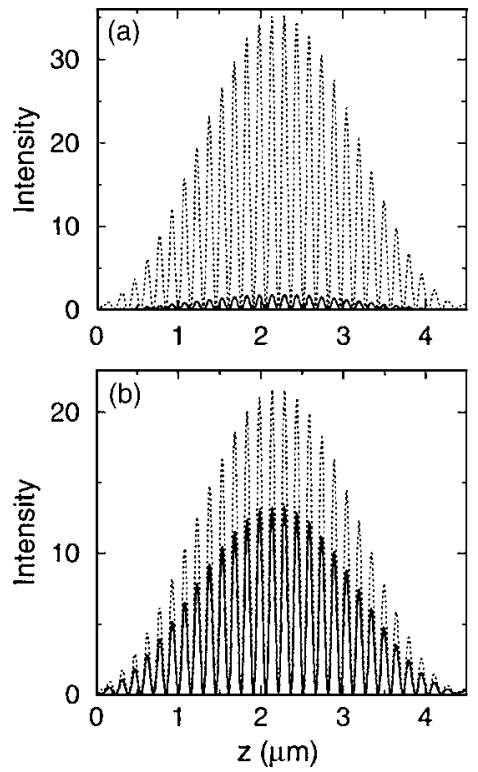

FIG. 4. Intensity distributions for components of wavelength $\lambda_{1}=681.3 \mathrm{~nm}$ (dotted curve) and wavelength $\lambda_{2}=680.32 \mathrm{~nm}$ (solid curve) at point $B$ (a) and at point $C$ (b) of Fig. 2. The intensity is normalized by $I_{1}(0)$.

ferent from the multistability found in the case of single incident beam, where different solutions correspond to soliton trains with different numbers of envelopes [8].

The multistabilities studied above were observed via the change of input intensity at a fixed set of operating wavelengths. Similarly, we can observe the multistability via the change of operating wavelengths at a fixed set of input intensities and frequency detuning $\delta=\omega_{1}-\omega_{2}=2 \pi c\left(1 / \lambda_{1}\right.$ $\left.-1 / \lambda_{2}\right)$. In Fig. 5 we show two typical results of $I_{2}(L) / I_{2}(0)$ versus $\lambda_{1}$ for $I_{2}(0)=3.675 \mathrm{MW} / \mathrm{cm}^{2}$ with $I_{1}(0)$ $=397 \mathrm{MW} / \mathrm{cm}^{2}$ and $I_{1}(0)=415 \mathrm{MW} / \mathrm{cm}^{2}$, where bistability and tristability are clearly seen.

The tristability phenomenon disappears when $\tau \ll 1 / \delta$ or $\tau \gg 1 / \delta$ as the imaginary parts of $\kappa_{\alpha}$ 's in Eqs. (6a)-(6d) become small and energy transfer can be ignored. In these limits, we only find bistability curves for each wavelength component, with one single-envelope solution. To see how large the deviation of $|\delta \tau|$ from unit induces the disappearance of the tristability phenomenon, we make calculations with the

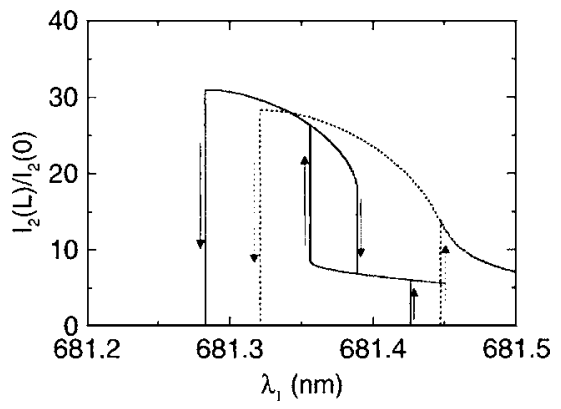

FIG. 5. $I_{2}(L) / I_{2}(0)$ as a function of $\omega_{1}$ in the superlattice of $N$ $=30$ unit cells. $I_{2}(0)=3.675 \mathrm{MW} / \mathrm{cm}^{2}$ and $\lambda_{1}-\lambda_{2}=0.98 \mathrm{~nm}$ which gives $|\delta \tau|=0.99$. Dotted curve: $I_{1}(0)=397 \mathrm{MW} / \mathrm{cm}^{2}$; solid curve: $I_{1}(0)=415 \mathrm{MW} / \mathrm{cm}^{2}$. 

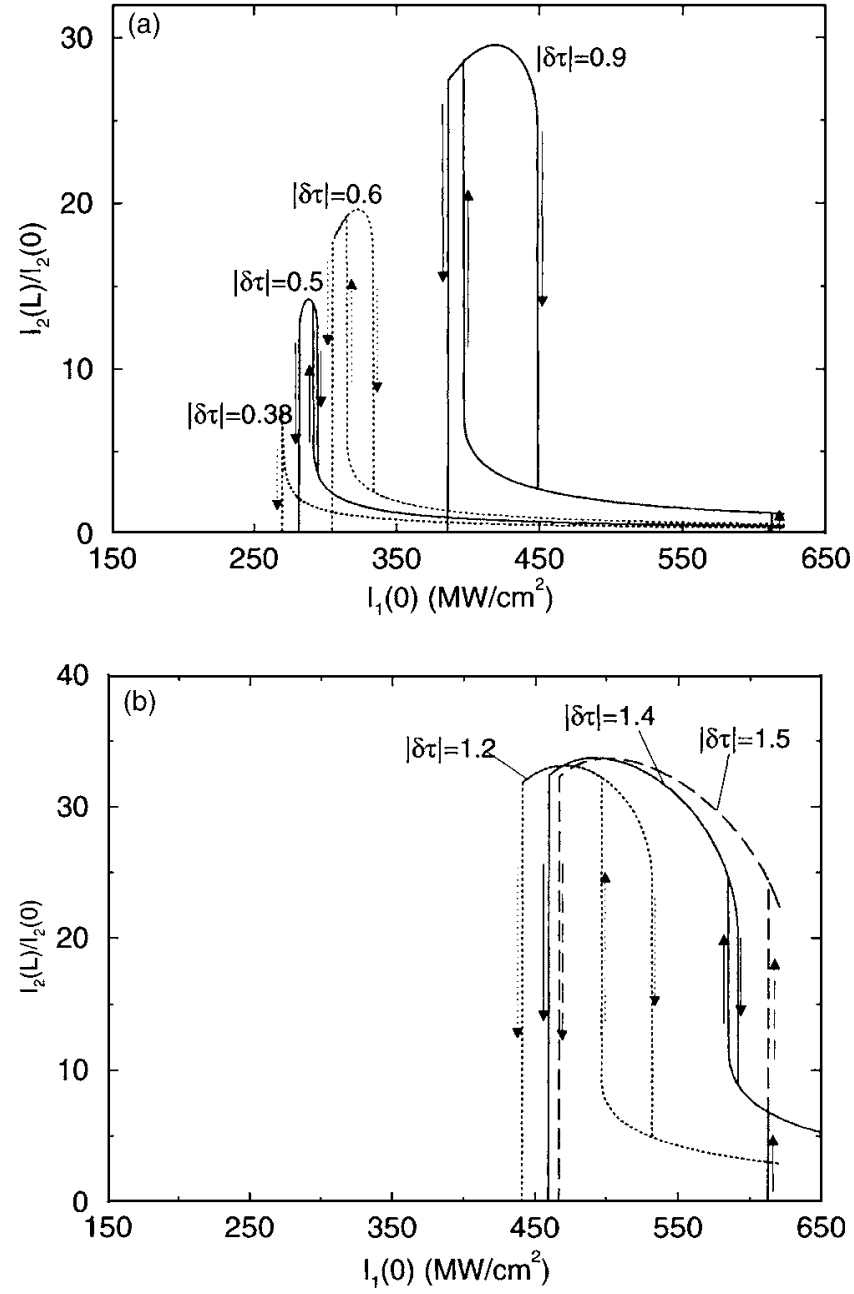

FIG. 6. (a) $I_{2}(L) / I_{2}(0)$ as a function of $I_{1}(0)$ for $\tau=228 \mathrm{fs}$ $(|\delta \tau| \approx 0.9), \quad \tau=152$ fs $(|\delta \tau| \approx 0.6), \quad \tau=126$ fs $(|\delta \tau| \approx 0.5)$, and $\tau$ $=96 \mathrm{fs}(|\delta \tau| \approx 0.38)$ in the superlattice of $N=30$ unit cells, with fixed $\lambda_{1}=681.3 \mathrm{~nm}$ and $\lambda_{2}=680.32 \mathrm{~nm}$. (b) $I_{2}(L) / I_{2}(0)$ as a function of $I_{1}(0)$ for $\tau=303$ fs $(|\delta \tau| \approx 1.2), \tau=354 \mathrm{fs}(|\delta \tau| \approx 1.4)$, and $\tau=379$ fs $(|\delta \tau| \approx 1.5)$.

same value of $\delta$, i.e., $\lambda_{1}=681.3 \mathrm{~nm}$ and $\lambda_{2}=680.32 \mathrm{~nm}$, but varying $\tau$. Similar to Fig. 2, in Fig. 6(a) we show $I_{2}(L) / I_{2}(0)$ versus $I_{1}(0)$, with fixed $I_{2}(0)=3.675 \mathrm{MW} / \mathrm{cm}^{2}$ for various value of $|\delta \tau|<1$. It is seen that, as $|\delta \tau|$ deviates from unit, the width of the tristability region becomes narrower and, as we have verified, when $|\delta \tau|<0.46$ the tristability disappears. For $|\delta \tau|>1$, we show some typical results in Fig. 6(b). Similar to the case of $|\delta \tau|<1$, the width of the tristability region becomes narrower as $|\delta \tau|$ increases from unit. When $|\delta \tau|$ $>1.45$, the tristability disappears. It is worth mentioning here that the limit of $\tau \ll 1 / \delta$ corresponds to the case studied in Ref. [3], where the strong pump beam $I_{1}$ tunes the frequency of the band-gap edge and the weak probe beam $I_{2}$ feels this frequency change.

\section{B. Enhancement of energy transfer by making use of defect state}

The above results show that the energy-transfer efficiency can be significantly enhanced through the formation of self-
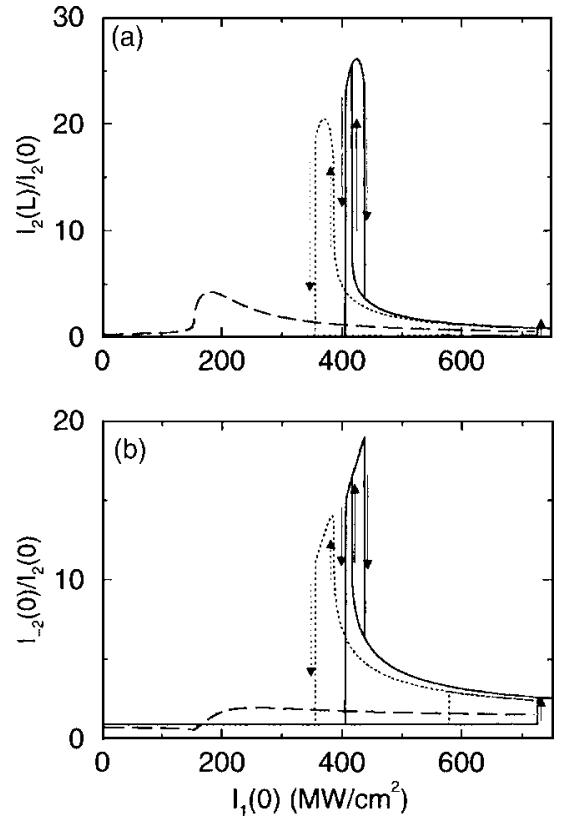

FIG. 7. $I_{2}(L) / I_{2}(0)$ (a) and $I_{-2}(0) / I_{2}(0)$ (b) as a function of $I_{1}(0)$ in the superlattice of $N=11$ unit cells with a defect. Dashed curves: $\lambda_{1}=673.95 \mathrm{~nm}, \lambda_{2}=672.97 \mathrm{~nm}$. Dotted curves: $\lambda_{1}=672.95 \mathrm{~nm}, \lambda_{2}$ $=671.97 \mathrm{~nm}$. Solid curves: $\lambda_{1}=672.75 \mathrm{~nm}, \lambda_{2}=671.77 \mathrm{~nm}$.

organized localized states near the band edge. Using the same principle, it is expected that large enhancement can also be achieved by use of the field localization in a defect state. To show this, we consider a superlattice of 21 layers, i.e., $11 \mathrm{CdS}$-nonlinear layers and $10 \mathrm{SiO}_{2}$-linear layers, with the width of each layer as $l_{1}=l_{2}=83 \mathrm{~nm}$ except the width of the middle CdS-nonlinear layer as $2 l_{2}=166 \mathrm{~nm}$. The transmission spectrum of the linear superlattice gives the defect modes occurring at $\lambda_{1 d}=675.183 \mathrm{~nm}$ and $\lambda_{2 d}=674.112 \mathrm{~nm}$ for the beam incident upon the sample at incident angles $\theta_{10}=2^{\circ}$ and $\theta_{20}=7^{\circ}$, respectively. Considering that the Kerr nonlinearity induces the shifts of the defect modes, we choose the operating wavelengths $\lambda_{1}$ and $\lambda_{2}$ slightly smaller than $\lambda_{1 d}$ and $\lambda_{2 d}$, respectively. We find that the output intensities exhibit the similar behaviors as those shown in Fig. 2, i.e., as $\lambda_{1}$ and $\lambda_{2}$ are moved away from $\lambda_{1 d}$ and $\lambda_{2 d}$ the output intensity versus input intensity changes from the single value to bistablity and then to tristability. For example, in Figs. 7(a) and 7(b) we show three typical results for $I_{2}(L) / I_{2}(0)$ and $I_{-2}(0) / I_{2}(0)$ versus $I_{1}(0)$, respectively, where we fix $I_{2}(0)=3.675 \mathrm{MW} / \mathrm{cm}^{2}$. Dashed curves are for $\lambda_{1}$ $=673.95 \mathrm{~nm}$ and $\lambda_{2}=672.97 \mathrm{~nm}$, dotted curves for $\lambda_{1}$ $=672.95 \mathrm{~nm}$ and $\lambda_{2}=671.97 \mathrm{~nm}$, and solid curves for $\lambda_{1}$ $=672.75 \mathrm{~nm}$ and $\lambda_{2}=671.77 \mathrm{~nm}$. The wavelengths used in Fig. 7 give $|\delta \tau|=0.99$. From the calculated value of $\left[I_{2}(L)\right.$ $\left.-I_{2}(0)\right] / I_{2}(0) \approx 2.1 \times 10^{-3}$ for $I_{1}(0)=181 \mathrm{MW} / \mathrm{cm}^{2}$ in a homogeneous medium of the same $n_{2}$ and $L=1.743 \mu \mathrm{m}$, we see from Fig. 4 that the energy-transfer efficiency is enhanced by more than three orders of magnitude.

\section{CONCLUSION}

In conclusion, we show that the energy-transfer efficiency by nondegenerate two-beam coupling in a $1 \mathrm{D}$ Kerr-nonlinear 
superlattice can be enhanced by several orders of magnitude. Such an effective enhancement is due to the excitation of the coupled localized state with two wavelength components. The efficiency can also be enhanced significantly by utilizing the shifted coupled defect modes. Due to the intensityinduced index modulation [4], the bistability is observed, and because of the energy transfer between different wavelength components a state can occur, which results in a tristability behavior. Finally, since the sample thicknesses used in this work are 4.5 and $1.743 \mu \mathrm{m}$, our work presents a way toward high-efficiency and microsized optical devices for optical signal amplification and optical interconnection.

\section{ACKNOWLEDGMENTS}

The authors would like to thank K. S. Wong for many useful discussions. This work was supported by Hong Kong RGC Grant No. 605804.
[1] See, for example, C. M. Bowden and A. M. Zheltikov, J. Opt. Soc. Am. B 19, 2046 (2002), and references therein.

[2] M. Scalora et al., Phys. Rev. A 56, 3166 (1997); Opt. Photonics News 12(4), 38 (2001); A. V. Andreev et al., J. Opt. Soc. Am. B 19, 2083 (2002); A. M. Malvezzi et al., ibid. 19, 2122 (2002).

[3] M. Scalora et al., Phys. Rev. Lett. 73, 1368 (1994); P. Tran, Opt. Lett. 21, 1138 (1996).

[4] B. Xu and N. B. Ming, Phys. Rev. Lett. 71, 1003 (1993); 71, 3959 (1993); Z. L. Wang et al., Phys. Rev. B 53, 6984 (1996).

[5] E. Centeno and D. Felbacq, Phys. Rev. B 62, R7683 (2000).

[6] W. Chen and D. L. Mills, Phys. Rev. Lett. 58, 160 (1987).

[7] C. M. de Sterke and J. E. Sipe, in Progress in Optics XXXIII, edited by E. Wolf (Elsevier, Amsterdam, 1994), Chap. III; S. John and N. Akozbek, Phys. Rev. Lett. 71, 1168 (1993); N. Akozbek and S. John, Phys. Rev. E 57, 2287 (1998); S. F.
Mingaleev and Y. S. Kivshar, Phys. Rev. Lett. 86, 5474 (2001).

[8] P. Xie, Z. Q. Zhang, and X. Zhang, Phys. Rev. E 67, 026607 (2003); 69, 036601 (2004).

[9] P. Xie and Z. Q. Zhang, Phys. Rev. Lett. 91, 213904 (2003).

[10] M. G. Martemyanov, T. V. Dolgova, and A. A. Fedyanin, J. Exp. Theor. Phys. 98, 463 (2004); P. P. Markowicz et al., Phys. Rev. Lett. 92, 083903 (2004); P. Xie and Z. Q. Zhang, Phys. Rev. E 71, 026610 (2005).

[11] P. Xie and Z. Q. Zhang, Phys. Rev. A 69, 053806 (2004).

[12] R. W. Boyd, Nonlinear Optics (Academic Press, New York, 1992) Chap. 6.4, pp. 269-274.

[13] W. Zhang, H. Wang, K. S. Wong, Z. K. Tang, G. K. L. Wong, and R. Jain, Appl. Phys. Lett. 75, 3321 (1999); [Here we have assumed that the $\chi^{(3)}$ response time of CdS is close to that of ZnO. K. S. Wong (private communication)]. 